\title{
A BIFURCATION THEOREM
}

\section{PAUL WALTMAN ${ }^{1}$}

1. This paper presents a bifurcation theorem for the second order system

$$
\begin{aligned}
& \frac{d x_{1}}{d t}=f_{1}\left(x_{1}, x_{2}, \mu\right), \\
& \frac{d x_{2}}{d t}=f_{2}\left(x_{1}, x_{2}, \mu\right) .
\end{aligned}
$$

A description of the classical bifurcation problem will be found in Minorsky [1, Chapter V] and Andronov and Chaikin [2, Chapter 6]. Here the functions $f_{1}$ and $f_{2}$ are required to be analytic and the proof depends on the series expansion guaranteed by the analyticity.

In [3, Chapter IV, §6], K. O. Friedrichs establishes a bifurcation theorem which does not require analyticity but uses the implicit function theorem. Continuous second derivatives are required.

Since the differentiability conditions are less severe, the theorem presented here could apply when the theorem of Friedrichs does not. However, the purpose of the paper is to show the bifurcation process geometrically - to show that it arises from the properties of trajectories considered as point sets and from the continuity of the vector field. To this end, the existence of a certain dynamical system will be postulated. This is in contrast to the above mentioned bifurcation theorems where conditions are prescribed (on certain coefficients, matrices, etc.) to guarantee the existence of an appropriate system. This will show the dependence of the theorem on the structure of the dynamical system rather than on analytical conditions necessary to achieve it.

2. A critical point $p=\left(p_{1}, p_{2}\right)$ in the plane is said to be a stable (unstable) spiral point if there is a region $R$ such that the trajectory $\left(\phi_{1}(t), \phi_{2}(t)\right)$ through any point of $R$ has the properties that (i) $\left(\phi_{1}(t), \phi_{2}(t)\right) \rightarrow\left(p_{1}, p_{2}\right)$ as $t \rightarrow \infty(t \rightarrow-\infty)$ and (ii) that the function $w(t)=\tan ^{-1}\left(\phi_{2}(t) / \phi_{1}(t)\right)$ is such that $|w(t)| \rightarrow \infty$ as $t \rightarrow \infty(t \rightarrow-\infty)$ [4, p. 376]. It will be assumed that the region $R$ above has been extended to a canonical region in the sense of Markus [5]. The solution curves which bound these regions are called separatrices. For a precise defini-

Received by the editors March 4, 1963.

1 This work was performed under the auspices of the United States Atomic Energy Commission. 
tion and for properties of separatrices the reader is referred to [5].

The phase space associated with (1) can be thought to be a "stack" of planes by adding an equation $\mu^{\prime}=0$. It will be convenient here, however, to project each $\mu$-plane onto a single plane such that for any choice of $\mu$ we have a family of associated solution curves. For a fixed $\mu$, we shall denote by $R_{\mu}$ the canonical region associated with a spiral point plus the critical point itself.

Theorem. Suppose that (i) in equations (1) $f_{1}$ and $f_{2}$ are continuous in $E^{3}$ and for a fixed $\alpha$ are $C^{\prime}$ in $(x, y)$, (ii) there is an interval I containing a point $\mu_{0}$ such that for $\mu$ in I and a critical point $p, R_{\mu}(p)$ is simply connected and $p$ is a stable spiral point for $\mu<\mu_{0}$ and is an unstable spiral point for $\mu \geqq \mu_{0}$, (iii) $\mu$ appears in (1) so as not to affect the location of critical points in the plane, (iv) all critical points are isolated. Then there is an interval $\left(\mu_{1}, \mu_{0}\right)$ such that for $\mu_{1}<\mu<\mu_{0}$ there is a limit cycle surrounding $p$ which tends to $p$ as $\mu$ tends to $\mu_{0}$. If in condition (ii) the words stable and unstable are interchanged or the inequalities are reversed, a similar theorem is valid.

Choose a monotone increasing sequence $\left\{\mu_{n}\right\}$ which converges to $\mu_{0}$. To avoid double subscripts let $R_{\mu_{n}}$ be denoted by $R_{n}$. Let $\bar{R}_{n}$ denote the closure of $R_{n}$.

LEMMA. $\lim \bar{R}_{n}=p$.

It will be shown first that $\lim R_{n}=p$. Since $p$ is in each $R_{n}, p$ is in $\lim \inf R_{n}$. Suppose $\lim \sup R_{n}$ contains a point $q=\left(q_{1}, q_{2}\right), q \neq p$. Since $q$ is in infinitely many $R_{n}$, choose a subsequence, denoted again by $R_{n}$, such that $q$ is in each $R_{n}$. Denote the solution through $q$ at $t=0$ as $\left[x_{1}\left(t, q, \mu_{n}\right), x_{2}\left(t, q, \mu_{n}\right)\right]$ and let $L$ be the line joining $p$ and $q$. Since, by hypothesis, each of the above solutions is a stable spiral, the trajectory must cut $L$ at a first time $t_{n}$, on the $p$ side of $q$. Suppose $q \in R_{\mu 0}$. If $t_{n} \rightarrow \infty$, then the trajectory $\left[x_{1}\left(t, q, \mu_{0}\right), x_{2}\left(t, q, \mu_{0}\right)\right]$ is stable in the sense of Poisson (and hence periodic) which contradicts the hypothesis that $\left[x_{1}\left(t, q, \mu_{0}\right), x_{2}\left(t, q, \mu_{0}\right)\right]$ is in $R_{\mu 0}$. Since the trajectory $\left[x_{1}\left(t, q, \mu_{0}\right), x_{2}\left(t, q, \mu_{0}\right)\right]$ is in an unstable spiral region, it must cut the line $L$ on the non- $p$ side of $q$, say at distance $d$ from $q$. Since $\left\{t_{n}\right\}$ have an upper bound $T$, the above situation violates the continuity in the initial conditions guaranteed by the smoothness of $f_{1}$ and $f_{2}$.

The assertion will be complete if we show that if there is a $q \in \lim \sup R_{n}$, and $q \notin R_{\mu 0}$, then there is a $q^{*} \in \lim$ sup $R_{n}$ such that $q^{*} \in R_{\mu 0}$. Let $D$ be a closed circular disc containing $p$ and lying interior to $R_{\mu 0}$. Since the points of $D$ may not be in $\lim \sup R_{n}$, then for large $n$, separatrices must pass arbitrarily close to $p$ (or else the asser- 
tion is readily established) and hence must cut the boundary of $D$. For each $n$ choose $r_{n}$ as intersection of a separatrix directed interior to $D$ and the boundary of $D$. An arc of the boundary of $D$ of which $r_{n}$ is either the left or right endpoint, lies in $R_{n}$. The arc may be lengthened until a second intersection of a separatrix and the boundary of $D$ is encountered. Denote this point by $s_{n}$; necessarily the vector field is directed from inside to outside $D$, since the arc joining $r_{n}$ and $s_{n}$ lies in a spiral region. Since the boundary of $D$ is compact, the sequence $\left\{r_{n}\right\}$ has a limit point $r$; choose a subsequence $\left\{r_{n}^{\prime}\right\}$ converging to it; let $\left\{s_{n}^{\prime}\right\}$ denote the corresponding subsequence of $\left\{s_{n}\right\}$. This subsequence has a limit point $s$, choose $\left\{s_{n}^{\prime \prime}\right\}$ converging to it. If $r=s$, then there is a discontinuity in the vector field, for at each $r_{n}$ the vector is "inbound" and at $s_{n}$, "outbound." Tangency is not possible since the trajectories pass arbitrarily close to $p$. If $r \neq s$, then one can find an arc (a subarc of the arc joining $r$ and $s$ ) which lies in infinitely many $R_{n}$. Let $q^{*}$ be any point of this arc. This completes the proof of the assertion that $\lim R_{n}=p$.

Now suppose $\lim \sup \bar{R}_{n}$ contains a point $q \neq p$. First it will be supposed that $q$ is noncritical. By the preceding paragraph $q$ may be in at most a finite number of the $R_{n}$, hence it is in infinitely many $\bar{R}_{n}-R_{n}$, that is, $q$ lies on the boundary of $R_{n}$ for infinitely many $R_{n}$. Select such a subsequence which will again be denoted by $\left\{R_{n}\right\}$. Let $D$ be a closed disc about $q$ of radius $\epsilon$, where $\epsilon$ is chosen small enough such that no critical point is in $D$. The boundary of each $R_{n}$ consists of separatrices, thus for each $n$ there is a separatrix through $q$. Since $D$ is noncritical, by the Poincaré-Bendixson Theorem the positive and negative half trajectories must leave $D$. Thus for each $n$ there are points $q_{n}$ and $q_{n}^{\prime}$ where the trajectory intersects the boundary of $D$. Let the vector field be "inbound" (i.e., crosses from outside to inside $D$ ) at $q_{n}$ and "outbound" (i.e., crosses from inside to outside $D$ ) at $q_{n}^{\prime}$. An open arc of the boundary of $D$ joining $q_{n}$ and $q_{n}^{\prime}$ lies in $R_{n}$. The set $\left\{q_{n}\right\}$ is finite or has an accumulation element $q^{*}$ on the boundary of $D$. Select a subsequence converging to $q^{*}$ or if the set was originally finite let $q^{*}$ be an element which was repeated infinitely often. Let $\left\{q_{n}^{\prime}\right\}$ be the subsequence of the corresponding "outbound" points and select a subsequence which converges to $q^{\prime}$ (or let $q^{\prime}$ be an element repeated infinitely often). If $q^{*} \neq q^{\prime}$ an arc of the boundary of $D$ can be selected that lies in infinitely many $R_{n}$ and this is impossible. If $q^{*}=q^{\prime}$ the vector field has a discontinuity at this point which is also impossible. (Note that the possibility of tangency is ruled out since each trajectory goes through $q$ and $q$ is positive distance from the boundary of $D$.) 
If $q$ is critical, then the boundary of $R_{n}$ for large $n$ consists of at least two paths which have $q$ as an $\alpha$-or $\omega$-limit point. Since the region which they bound is spiral, these trajectories are oppositely oriented. The proof can then proceed as above.

Thus $\lim \bar{R}_{n}=p$ and the lemma is established.

Proof of The Theorem. The idea of the proof is to establish that, for large $n$, the boundary of $R_{\mu}$ consists of a single separatrix which as a point set collapses to $p$ as $n \rightarrow \infty$. Choose a sequence $\left\{\mu_{n}\right\}$ such that $\mu_{n}$ tends to $\mu_{0}$ monotonically from below. Denote the region $R_{\mu_{n}}$ by $R_{n}$ as before. Let $C$ be a circle with center $p$ and radius $\epsilon$, where $\epsilon$ is sufficiently small to insure that no critical points other than $p$ lie inside or on $C$ for $\mu$ in some neighborhood of $\mu_{0}$. Suppose the separatrices forming the boundary of $R_{n}$ do not have $\omega$-limit points inside or on $C$ for large $n$-hence the trajectory forming the boundary of $R_{n}$, for large $n$, must cross $C$ once with the vector field "inbound" and once "outbound."

For each large $n$ select a point $q_{n}$ which lies on the intersection of a separatrix and $C$ with the vector field outbound. Proceed along $C$ within the region $R_{n}$ (i.e., on the $p$-side of the above separatrix) until a second intersection $q_{n}^{\prime}$ of $C$ and a separatrix which bounds $R_{n}$ is reached. Necessarily, the vector field at $q_{n}^{\prime}$ is inbound since the arc joining $q$ and $q_{n}^{\prime}$ lies in a spiral region. Let $r_{n}$ be the length of this arc. If $\lim \sup r_{n}$ is not zero, the above lemma is contradicted. Choose initial conditions $\left(q_{n}, \mu_{n}\right)$ and $\left(q_{n}^{\prime}, \mu_{n}\right)$. Through each of these, for each large $n$, is a trajectory "inbound" and "outbound," respectively, and passing arbitrarily close to $p$. Since $C$ is a compact point set, it is possible to choose a convergent subsequence of $\left\{q_{n}\right\}$, and since $\lim r_{n}$ $=0$, the corresponding subsequence of $\left\{q_{n}^{\prime}\right\}$ converges to the same limit. Thus there is a discontinuity in the vector field, which is the desired contradiction. Hence for large $n$ some separatrices have $\omega$ limit points inside $C$. Since the curves are separatrices, and $p$ is a spiral point, the $\alpha$ - or $\omega$-limit points must be different from $p$, and thus can only be a point of the trajectory itself. Other than critical points the only "stable-à-la-Poisson" trajectories in the plane are closed curves [6, Chapter 2] which establishes the existence of a periodic solution.

By the lemma, these closed trajectories must tend to $p$ as $n \rightarrow \infty$.

3. An important physical example of a system to which the above may apply (under appropriate hypotheses on $f$ and $q$ ) is

$$
\frac{d x_{1}}{d t}=\mu x_{1}\left[k_{1}-x_{1}-f\left(x_{2}\right)\right], \quad \frac{d x_{2}}{d t}=x_{2}\left[k_{2}-x_{2}-g\left(x_{1}\right)\right] .
$$


This system is used to describe the growth of competing biological populations and is analyzed in detail in [7] and [8].

\section{REFERENCES}

1. N. Minorsky, Introduction to non-linear mechanics, J. E. Edwards, Ann Arbor, Mich., 1947.

2. A. Andronov and C. E. Chaikin, Theory of oscillations, Princeton Univ. Press., Princeton, N. J., 1949.

3. K. O. Friedrichs, Advanced ordinary differential equations, New York University Lecture Notes, 1949.

4. E. A. Coddington and N. Levenson, Theory of ordinary differential equations, McGraw-Hill, New York, 1955.

5. L. Markus, Global structure of ordinary differential equations in the plane, Trans. Amer. Math. Soc. 76 (1954), 127-148.

6. V. V. Nemickii and V. V. Stepanov, Qualitative theory of differential equations, Princeton Univ. Press, Princeton, N. J., 1960.

7. W. S. Cunningham, Simultaneous nonlinear differential equations of growth, Bull. Math. Biophys. 17 (1955), 101-110.

8. W. R. Utz and P. Waltman, Periodicity and boundedness of solutions of generalized differential equations of growth, Bull. Math. Biophys. 25 (1963), 75-93.

Sandia laboratory, Albuqueroue 